\title{
Cambio tecnológico, migración de talento y habilidades de la fuerza laboral en el sector manufacturero en México y el mundo (2015-2019)
}

Mario Alberto Morales Sánchez*

Partiendo del marco teórico de la economía evolutiva, en este trabajo se realiza un análisis sobre la migración de talento laboral entre las distintas subramas de la industria manufacturera y las habilidades laborales que requiere el mercado en cada una de ellas. Se realiza una comparación del caso mexicano con el mundo, para determinar cuáles son las principales ramas atractoras y expulsoras de trabajo, así como las habilidades laborales más demandadas en cada rama. Para ello se utilizan datos proporcionados por el Banco Mundial en alianza con LinkedIn, Digital Data for Development, y se emplea la teoría de grafos como metodología para el análisis de estos. La principal aportación radica en establecer cuáles son las ramas manufactureras atractoras de empleo en México y a nivel mundial, y cuáles aquellas que han tenido una pérdida neta en los últimos 5 años. En términos prospectivos este análisis puede contribuir al desarrollo de políticas industriales enfocadas a las ramas dinámicas de la economía mundial.

Palabras clave: innovación tecnológica; capital humano; migración de talento; política industrial.

Clasificación JEL: 015, 025, O30.

* Profesor de tiempo completo, Facultad de Economía, UNAM, México.

Correo-e: almoralesanchez@gmail.com 


\section{ABSTRACT}

\section{Technological change, migration of talent and skills of the workforce in the manufacturing sector in Mexico and the world (2015-2019)}

Based on the theoretical framework of the evolutionary economy, this work presents an analysis on the migration of work talent between the different branches of the manufacturing industry, and the main skills demanded. A comparison of the Mexican case is made with respect to the world to determine which are the main industries of attraction and expulsion of work. This is done using data provided by the World Bank in partnership with LinkedIn, Digital Data for Development, using graph theory as a methodology for the analysis. The main contribution lies in establishing which are the manufacturing branches that are attracting employment in Mexico and globally, and those that have had a net loss in the last 5 years. In prospective, this analysis could contribute to the development of industrial policies focused on the dynamic industries of the world economy.

Keywords: Technological innovation, Human capital, Talent migration, Industrial policy.

JEL Classification: 015, 025, O30.

\section{INTRODUCCIÓN}

La reconfiguración económica ocurrida en los últimos 20 años a partir de la propagación de los cambios tecnológicos relacionados con las tecnologías de la información y comunicación ha cambiado los patrones de especialización de las economías y las habilidades requeridas por parte de los trabajadores para desempeñar actividades tanto tradicionales como las de reciente aparición.

El presente trabajo analiza precisamente los cambios en los patrones de especialización laboral, medida a través de la migración de talento dentro del sector manufacturero y hacia afuera del mismo, así como las habilidades laborales requeridas actualmente en cada una de las ramas de dicho sector. Esta comparación se enfoca en México a partir de las principales tendencias mundiales.

Este estudio permite tener un panorama general de cómo los cambios tecnológicos recientes han impactado tanto la dinámica de 
crecimiento de las ramas de la industria manufactura, como las habilidades de la fuerza de trabajo para colocarse en actividades dentro o fuera de las manufacturas.

Para ello se utilizan datos de la alianza entre el Banco Mundial y LinkedIn, Digital Data for Development, que analizan la información de millones de suscriptores a LinkedIn para arrojar información relativa a la migración de talento entre países y sectores económicos, además de proveer información relacionada con la acelerada modificación de las habilidades de trabajo que las economías demandan. Estos datos son analizados con la metodología de teoría de grafos.

El análisis de la información permite observar patrones que muy probablemente reflejen tendencias de largo plazo en los mercados laborales, como la migración de talento de alta y mediana especialización de las economías de ingresos medios y bajos hacia economías desarrolladas. Ello tiene importantes implicaciones para las políticas de inversión en formación de capital humano en virtud de que, en una proporción creciente, el gasto en la etapa formativa de los futuros trabajadores se realiza en las economías en desarrollo, pero, ante la falta de dinamismo de los mercados laborales en estos países, se producen efectos de expulsión de talento hacia economías con mercados de trabajo de mayor dinamismo y mejores salarios.

De acuerdo con los datos de LinkedIn, en los últimos 5 años la mayoría de los países en desarrollo perdió talento neto que migró hacia países de altos ingresos. De las 20 naciones que más talento atrajeron, 14 eran de ingreso medio y alto, particularmente en sectores transables.

En parte, ello se debe al mayor dinamismo de los sectores tradicionales en los países de altos ingresos, pero también al mayor desarrollo en sectores de reciente creación y muy alto crecimiento, como los basados en conocimiento y con un estrecho vínculo con la actividad científica y tecnológica, como la nano y biotecnología, la robótica, la inteligencia artificial, etc.

Pero no sólo existe migración de talento entre países, sino que también el cambio tecnológico ha generado una reconfiguración de los flujos de talento al interior de las industrias en un mismo país. Los datos muestran que hay actividades económicas que en los últimos 5 años han expulsado talento hacia otras industrias, lo que, a su vez, ha incentivado una reconfiguración de las habilidades tecnológicas, cada vez más altas 
y especializadas, que la fuerza de trabajo requiere para desempeñarse exitosamente en las actividades hacia las que migran.

De esta forma, el estudio muestra el flujo de trabajadores entre las ramas de la manufactura, tanto en tendencias mundiales como en la forma que éstas adoptan, en el caso específico de México. Al respecto, las teorías de las capacidades o competencias (Hodgson, 2007), concretamente las vinculadas con la teoría evolutiva (Nelson y Winter, 1982; Antonelli, 2014; Harper, 2018) muestran, en última instancia, que son las capacidades y habilidades de la fuerza laboral las que moldean ambientes propicios para la capacidad innovadora en las economías.

Como es sabido, en las últimas décadas el sector manufacturero mexicano ha sido un enclave de generación de empleos, inversión y exportaciones principalmente. Sin embargo, trabajos previos que estudian la estratificación de la industria en México con base en teoría de grafos y análisis de matriz-insumo- producto, como el que presentan Fuentes y García (2009) y García, Morillas y Ramos (2005), encuentran que el sector manufacturero ha dejado de ser clave en la articulación del sistema económico si se consideran las relaciones de causalidad basadas en las interrelaciones productivas internas y globales, así como de la centralidad en el sistema. Una de las explicaciones posibles de esta pérdida de centralidad de la manufactura en el sistema económico es la falta de adaptación de las habilidades laborales a las nuevas condiciones tecnológicas presentes en el mercado mundial. En este sentido, el presente trabajo es complementario con los hallazgos presentados en los trabajos referidos.

Otra variable que puede ayudar a comprender la migración de talento es el nivel salarial. Mientras el promedio mensual de los salarios en América Latina en 2020 es de 443.33 dólares, el promedio salarial de los 20 países con mayores retribuciones salariales es de 338.05 dólares por mes. México se encuentra por encima de la media latinoamericana con 467 dólares. ${ }^{1}$ Por ejemplo, trabajos como el que presentan Patt et al. (2020) concluyen que las habilidades de los migrantes mexicanos en Estados Unidos representan una variable fundamental para determinar diferencias en términos salariales. Plantean que las habilidades ocupacionales proveen información muy parecida a la que se obtiene

\footnotetext{
${ }^{1}$ Organización Internacional del Trabajo $(2021)<\mathrm{https}$ ://ilostat.ilo.org $>$.
} 
del análisis con base en salarios, aunque esta última variable resulta una mejor aproximación para explicar la capacidad productiva de los trabajadores migrantes. Aunque la disparidad salarial contribuye con la migración de talento entre países, y resulta de interés desarrollar líneas de investigación que vinculen la migración laboral con el salarial, la base de datos utilizada para realizar esta investigación no permite establecer dicha relación, por lo que se concentra en la identificación de habilidades.

Este trabajo se divide de la siguiente manera: en la sección segunda se muestra el marco teórico relativo a la importancia de las capacidades y habilidades como factores de crecimiento económico. En la sección tres se muestran las características de los datos y la metodología que sirve como base del desarrollo de los elementos de análisis, concretamente, la teoría de grafos. En la sección cuarta se muestran los resultados y la interpretación de estos, para finalizar con las conclusiones y opciones de política industrial.

\section{MARCO TEÓRICO Y REVISIÓN DE LA LITERATURA}

En términos generales, la teoría económica concibe la innovación tecnológica como conocimiento acumulado por las empresas en un contexto de competencia, que permite la creación de nuevas soluciones técnicas (nuevos productos, servicios, procesos, etc.), las cuales generan ganancias extraordinarias en el largo plazo. Sin embargo, una diferencia importante entre distintas teorías se relaciona con quiénes son los portadores de ese conocimiento o, en otros términos, ¿el proceso de aprendizaje y conformación de capacidades ocurre en las organizaciones o en los individuos? Esta pregunta ha sido respondida dentro de la literatura especializada en al menos dos sentidos: en primer lugar, la llamada teoría económica evolutiva ha postulado mayoritariamente que los procesos de aprendizaje, creación retención y replicación de conocimiento ocurren dentro de las empresas como organización; en segundo lugar, otros autores han establecido que tales procesos ocurren prioritariamente a través de la interacción de los individuos.

Desde el punto de vista de la teoría económica, y en particular de la perspectiva de la teoría económica evolutiva, el aprendizaje ocurre a nivel de las empresas, por lo que es la estrategia más importante para la generación de nuevas combinaciones productivas que desencadenen 
procesos de innovación (Marengo y Pasquali, 2008). El aprendizaje genera la conformación de capacidades organizacionales que se utilizan no sólo para combinar de manera óptima los factores de la producción requeridos, sino principalmente para experimentar con nuevos insumos y procesos productivos que dinamizan y mejoran la producción. Si bien una empresa puede desarrollar un conjunto amplio de capacidades productivas, existe un subconjunto de ellas asociado directamente con el aprendizaje para la innovación. Las diversas teorías del cambio tecnológico basadas en esta concepción proponen que la generación de mejores combinaciones ocurre mediante un proceso de acumulación y combinación de conocimiento al interior de las empresas, expresado en la obtención sistemática de capacidades empresariales (Dosi et al., 2000).

Dicho conocimiento se produce a partir de la mejora continua de las prácticas productivas (Nelson y Winter, 1982), y la absorción de nuevo conocimiento generado por otras organizaciones (Cohen y Levinthal, 1990), sean estas otras empresas centros de innovación y desarrollo, etc. Todo este proceso de creación y absorción de conocimiento depende de lo que se ha llamado aprendizaje organizacional; es decir, el proceso mediante el cual los sujetos dentro de una organización adquieren y transforman la información interna y externa para generar patrones comunes de comportamiento y acción que se difunden y generalizan en toda la organización (Dosi et al., 2003).

Nelson y Winter (1982) proponen un énfasis especial en la importancia del conocimiento organizacional, como un atributo de la "empresa como un todo", el cual no se constituye como una suma o agregación simple del conocimiento de los agentes individuales, sino que representa un conocimiento distinto e identificable de manera independiente. Debido a que un sólo agente es incapaz de conocer todas las tareas que se realizan al interior de una empresa, la organización como un ente unificado "sabe" más que cualquier agente individual. Estos mismos autores establecen que el conocimiento adquirido por una organización puede ser modificado de manera deliberada por medio de esfuerzos conscientes dirigidos a alcanzar algún fin particular, aunque también es posible que se modifique por hechos no deliberativos, resultado de la acción automática de los agentes, o por factores azarosos que incluyen todo tipo de contingencias operacionales o medioambientales. Una de las aportaciones más importantes de la teoría evolutiva es haber 
reintroducido en el debate económico el concepto de rutinas organizacionales como una forma de explicar el funcionamiento de la empresa. Las rutinas son entendidas como habilidades cognitivas organizacionales que tienen la característica de ser programáticas, no deliberativas y automáticas.

Por otra parte, Cañibano y Potts (2019) establecen que es necesario reconstituir los fundamentos conceptuales de la teoría económica evolutiva al trasladar los fundamentos de la generación colectiva de conocimiento (en las empresas primordialmente) hacia una epistemología del agente individual que genera y utiliza el conocimiento en una estructura organizacional determinada. En el mismo sentido, Foss (2006) establece que la empresa, al igual que cualquier otra organización, se encuentra definida por la interacción de agentes individuales dentro de una unidad colectiva, la cual se sitúa en dos niveles distintos de agregación: el colectivo, que se expresa en las características derivadas de los fenómenos sociales; y el individual, definido por las acciones emprendidas por los agentes al interior de la empresa. Son precisamente estos agentes individuales los que tienen la capacidad biológica de aprendizaje mediante la creación, selección y retención de conocimiento. Por su parte, Vromen (2006a) establece que puede ser un error intentar reducir el conocimiento sólo a lo que ocurre dentro de la cabeza de los individuos: los aspectos sociales y culturales tienen una importancia decisiva en el proceso cognitivo. El problema es que la teoría económica evolutiva, al parecer, sólo reconoce un tipo de conocimiento social y tácito, que es automático y que aparentemente no tiene su origen en la consciencia individual de los agentes. Para estos autores resulta más conveniente recurrir a una concepción de agente empresarial que tome en cuenta la capacidad de los individuos para realizar acciones conscientes y deliberadas, como una manera de superar los problemas impuestos por la incertidumbre del ambiente. No obstante, esto no descarta que también pueda existir algún tipo de comportamiento no deliberativo y rutinario en ciertos procedimientos internos de la empresa.

Al respecto, Vromen (2006b) plantea que la deliberación consciente también presupone ciertas "habilidades" para su ejecución, que no todos los individuos poseen, por lo que el campo del comportamiento con base en habilidades no se encuentra restringido a un modo particular de comportamiento, más bien comprende todo el desempeño exitoso dentro de las empresas. Una regla que puede ayudar a enfrentar la incer- 
tidumbre es, por ejemplo: si ocurren situaciones del tipo $X$, entonces hacer $Y$; esto implica una elección consciente. No todas las elecciones conscientes se conducen de este modo; existen algunos casos en que los agentes se sienten suficientemente confiados para solucionar un problema sólo mediante la concentración en ese problema específico, y prefiguran su solución sin recurrir a reglas de deliberación consciente que hayan funcionado bien en situaciones previas (Vromen, 2006b).

La acción individual dentro de las organizaciones no debe interpretarse únicamente como una especie de reglas inconscientes que determinan la acción (rutinas), sino también como "pistas" o "claves" que desencadenan en el agente un proceso consciente de transformación de la información en decisiones que le permiten actuar en situaciones novedosas. El punto central es que existe una "coexistencia" permanente al interior del individuo entre las acciones derivadas de su elección consciente y las que son inconscientes o rutinarias; es decir, en algunas ocasiones las actividades automáticas se encuentran precedidas de una elección consciente.

No obstante, debe notarse que desde ambas perspectivas las habilidades o capacidades individuales anteceden la acción colectiva. Si bien para la teoría económica evolutiva las habilidades individuales no se explican sin situarse o contextualizarse dentro de un conjunto de acciones colectivas convertidas en rutinas, debe entenderse que la base del funcionamiento organizacional son precisamente dichas habilidades que el funcionamiento colectivo logra armonizar con las estrategias y objetivos de la empresa. Respecto a las habilidades individuales requeridas para el funcionamiento de las rutinas, si bien pueden adquirirse previamente, para la teoría económica evolutiva son más importantes para el funcionamiento de la organización aquellas que se conforman en los centros directos de actividad. Autores como los citados han flexibilizado los supuestos de la conducta no deliberativa y rutinaria presentes en la teoría económica evolutiva; han puesto mayor énfasis en el aprendizaje individual y en la obtención consciente de habilidades que ayuden a la solución de los problemas colectivos. En todo caso, la adquisición de habilidades individuales y colectivas se sitúa en el centro de la discusión relacionada con los factores que inciden en la emergencia de la innovación tecnológica, por lo que la adquisición y mejoramiento de dichas habilidades reviste una importancia de primer orden. 
Una posible solución a la discusión de la posición de las habilidades individuales en la dinámica y estructura de las organizaciones se encuentra en la síntesis propuesta por algunos autores al intentar responder la siguiente pregunta: ¿Cuál es la relación entre procesos cognitivos individuales y generación de reglas sociales? Dichos autores proponen un proceso evolutivo de generación de reglas que parte de lo individual y llega a lo social, como un proceso de construcción de modelos mentales colectivos con características emergentes. Esta propuesta se explica a continuación.

El planteamiento anterior concuerda con la explicación ofrecida por Dopfer y Potts (2008) sobre el fundamento del cambio organizacional evolutivo, al hacer explícito un elemento importante que consiste en proponer un proceso evolutivo capaz de explicar la emergencia de nuevas reglas en los agentes individuales y su adopción por la comunidad. Las reglas de segundo orden, o reglas diseñadas para generar nuevas reglas (las cuales son el fundamento evolutivo del cambio organizacional), tienen un proceso que comienza cuando un agente "inventa" una nueva forma de resolver un problema. Posteriormente, continúa con su adopción individual, cuando el agente logra constatar que dicha regla le permite una mejor solución al problema que enfrenta, y encuentra conveniente su utilización futura. El proceso finaliza con la retención de la regla a partir de su utilización reiterada en un conjunto de problemas específicos.

Sin embargo, para lograr que una o varias reglas individuales se consoliden y pueda generarse a partir de ellas una trayectoria organizacional específica es necesario que se generalicen en una población de carácter meso-económico, por lo que la fase de adopción individual, marcada como el momento en el que la nueva regla aparece ante la percepción de los demás agentes, se convierte en la fase de origen de una nueva regla social. Posteriormente, una vez que la nueva regla comienza a ser parte de la información disponible para los demás agentes, ocurre un proceso de replicación donde aquellas reglas que resulten exitosas serán adoptadas por cada vez más agentes como parte

\footnotetext{
La emergencia de una nueva regla obedece al proceso cognitivo en el cual el agente individual procesa cierta información procedente del medio ambiente y altera los esquemas mentales que ha logrado construir previamente, al corregir, reforzar o desaprender parte del conocimiento previo. Lo que aquí se propone es que, de esa combinación de información nueva con información pasada, surgen nuevas reglas de acción con distintas potencialidades de generalización.
} 
de sus acciones corrientes. Finalmente aparece también un proceso de retención donde lo que otrora fue una novedad "ideada" por un agente particular se institucionaliza y se convierte en una práctica socialmente aceptada. Este es un enfoque evolutivo del avance del conocimiento a través de la evolución de las reglas (Dopfer y Potts, 2008; Dopfer et al., 2004; Dopfer, 2005). En términos de la discusión que se ha dado hasta aquí puede afirmarse que la replicación de las reglas ocurre mediante la interacción de los agentes que participan en un contexto organizacional, por lo que su institucionalización como práctica recurrente es una manera de asegurar su autoejecución. La fase de institucionalización representa también una manera de concreción de nuevas expectativas entre los agentes, las cuales sustituyen dominios de interacción específicos.

En términos generales puede resumirse que el fundamento evolutivo del cambio en una organización se encuentra en el agente individual con capacidades cognitivas y de procesamiento de la información que le permiten transformar la información recabada del ambiente en nuevas reglas de acción que determinan su praxis cotidiana. Dichas reglas son de alguna manera validadas por la estructura social particular en la que el agente se desenvuelve, y su propagación depende de las interacciones que dinamizan a la población. En términos de la estructura empresarial, la adopción de nuevas reglas depende de la división cognitiva interna del trabajo y de las formas de gobierno que prevalezcan en un momento determinado; habrá estructuras de gobierno más inclinadas a la exploración del medio ambiente, por lo que su propensión al cambio será mayor que aquellas empresas poco expuestas a los parámetros externos. La adopción social de nuevas reglas de acción se erige como una concreción institucional, un patrón emergente de comportamiento con características propias. Esta nueva forma organizacional representa un parámetro agregado de estabilidad cognitiva e institucional, que condiciona las expectativas de los agentes individuales. 


\section{DATOS Y METODOLOGÍA}

\subsection{Datos}

Como se ha dicho, para este trabajo se utilizan datos provenientes de la colaboración entre el Banco Mundial (BM) y la empresa LinkedIn, Digital Data for Development. Esta alianza dio como resultado la recopilación de información de más de 100 países con al menos 100000 miembros de LinkedIn cada uno, distribuidos en 148 industrias y 50000 categorías de habilidades, lo cual representa una muestra no probabilística de al menos 10 millones de observaciones. Esta base datos presenta importantes ventajas en relación con otras fuentes de información, dado que capta la dinámica constante en el cambio de habilidades, que muchas ocasiones las encuestas gubernamentales tradicionales no capturan (Zhu et al., 2018).

Se utilizan las bases de migración de talento entre industrias ${ }^{3} \mathrm{y}$ de habilidades requeridas por la industria ${ }^{4}$ de los años 2015 a 2019 como insumos para el uso de las metodologías que se describen a continuación.

La utilización de este tipo de bases de información presenta la ventaja de captar en tiempo real la migración de talento entre las distintas ramas de la industria manufacturera en las áreas que conforman la base, en función de las habilidades requeridas por la industria, por lo que representan un indicador dinámico de las modificaciones impuestas por el cambio tecnológico en la composición de la fuerza laboral y de los polos de atracción de la misma. La desventaja evidente es que los datos no provienen de un muestreo probabilístico, por lo que la generalización de los resultados puede estar sujeta a cuestionamientos. No obstante, debido a que el número de observaciones es muy grande, que cada vez son más los usuarios que utilizan este tipo de plataformas digitales para colocarse en un puesto de trabajo y que la fuente de información

$3^{\circ} \quad$ Migración de habilidades: habilidades ganadas y perdidas. Según las habilidades asociadas con el perfil de un miembro en el momento de la migración. La ganancia o pérdida neta de miembros de otro país con una habilidad determinada dividida por la cantidad de miembros de LinkedIn con esa habilidad en el país objetivo (o seleccionado), multiplicado por 10000.

4 La métrica de habilidades requeridas por la industria captura qué habilidades tienen más probabilidades de agregarse al perfil de un miembro en una industria en comparación con otras industrias. Se calcula utilizando una versión adaptada de una técnica de minería de texto llamada Frecuencia de términos. Este método da más peso a una habilidad para una industria si más miembros de la industria enumeran la habilidad en sus perfiles y la habilidad es más exclusiva de la industria. Las habilidades incluidas son las que se agregan mientras un miembro tiene una ocupación particular (es decir, el enfoque de flujo de habilidades). 
permite realizar comparaciones internacionales, es posible conferir una validez analítica a este trabajo.

\subsection{Teoría de grafos}

En las últimas décadas la aplicación de la teoría de redes o grafos en las ciencias sociales ha cobrado gran importancia debido a que con ella se pueden analizar relaciones e interdependencias existentes entre conjuntos de datos, por ejemplo, países, individuos, empresas, sectores económicos, campos semánticos, etc. El comportamiento agregado de una economía no puede analizarse a partir de la interacción de individuos aislados, como propone la teoría neoclásica. Debido a que existe una interacción constante entre los agentes que conforman el sistema económico y que las acciones individuales tienen repercusión en el conjunto de los demás agentes, es posible representar el sistema en su conjunto como una red que evoluciona constantemente.

La evolución de las redes es un fenómeno endógeno, resultado de la interacción de los agentes con sus vecinos cercanos con base en la experiencia, lo que les permite aprender y adaptarse, por lo que se modifica la estructura de la red (Michel y Battiston, 2009). El análisis de redes en la ciencia económica ha sido poco explorado; sin embargo, recientemente ha tenido un importante auge debido a que este tipo de análisis cualitativo permite visualizar de manera sencilla estructuras complejas, como, por ejemplo, el diseño institucional y funcional de un sector o de una economía. En el análisis de una red se considera la estructura de las relaciones en las que cada actor (nodo) se encuentra involucrado; estos actores o nodos se describen a través de sus conexiones, las cuales si son relevantes para la estructura de los nodos, se visualizan en función de dicha relevancia (García, Morillas y Ramos, 2005).

Así, el análisis en red o grafos se centra en las relaciones entre entidades y no en las entidades por sí mismas. De forma sintética, una red es un conjunto de puntos que unidos mediante enlaces (líneas o aristas) o partir de una regla de asociación, que indica cómo se relacionan los nodos (Michael y Battiston, 2009). 


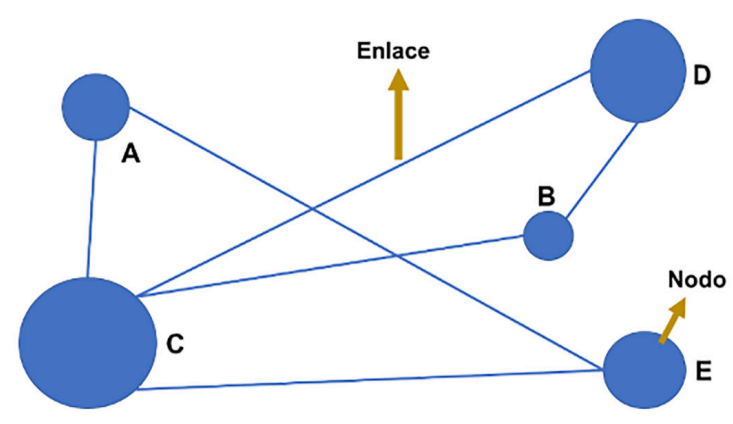

Fuente: elaborada con base en Newman, 2010.

Una red se puede denotar de manera estándar de la forma:

$$
G=(N, E)
$$

Donde $N$ es el conjunto de nodos y E es el conjunto de enlaces. Por lo tanto, la Figura 1 está compuesta por 5 nodos y 6 enlaces que pueden ser expresados como:

$$
\begin{gathered}
N=\{A, B, C, D, E\} \\
E=\{(A, C),(B, C),(C, D),(C, E),(D, B),(E, A)\}
\end{gathered}
$$

Una manera alternativa de representar la relación descrita en la red de la Figura 1 es con una matriz $W$, conocida como matriz de adyacencia o binaria. En esta matriz $W$ se representan los nodos en las filas y columnas. Si el elemento i está enlazado al elemento j, entonces los elementos $w_{i j} \mathrm{y} w_{j i}$ tendrán el valor de 1 . En caso contrario, si el vínculo es inexistente, el componente de la matriz tendrá un valor de cero; lo anterior tiene la implicación inmediata de que la matriz de adyacencia es simétrica; tiene el mismo número de filas que de columnas.

$$
W_{i j}=\left\{\begin{array}{c}
1, \text { si } i, j \text { están en lazados } \\
0, \text { si } i, j \text { no están enlazados }
\end{array}\right\}
$$




$$
\begin{gathered}
i, j=1, \ldots, n \\
W=\left[\begin{array}{lllll}
0 & 0 & 1 & 0 & 1 \\
0 & 0 & 1 & 1 & 0 \\
1 & 1 & 0 & 1 & 1 \\
0 & 1 & 1 & 0 & 0 \\
1 & 0 & 1 & 0 & 0
\end{array}\right] \\
W_{i j}=W_{j i}
\end{gathered}
$$

Un tipo distinto de análisis de redes puede establecerse mediante las redes o grafos ponderados, el cual muestra los enlaces de manera valuada o ponderada (Newman, 2010). Dichos enlaces se encuentran asociados a una intensidad, representada por un valor numérico; entre mayor es la intensidad de la asociación, mayor será también la representación, medida en anchura del vínculo entre los nodos. Una representación de lo anterior se muestra en la Figura 2 (Díaz, Morales y Cabrera, 2019).

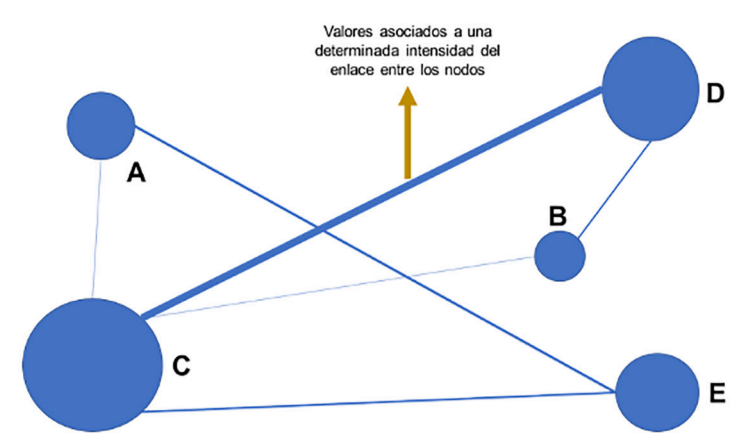

Fuente: elaborada con base en Mitchell (2009).

El grosor de los enlaces de la Figura 2 muestra la intensidad de la relación existente entre dos nodos. La representación matricial de la red anterior es: 


$$
\begin{aligned}
& Z=\left[\begin{array}{ccccc}
0 & 0 & 6 & 0 & 10 \\
0 & 0 & 1 & 5 & 0 \\
6 & 1 & 0 & 4 & 2 \\
0 & 5 & 4 & 0 & 0 \\
10 & 0 & 2 & 0 & 0
\end{array}\right] \\
& D_{c}=\sum_{j=1}^{n} x_{i j}=\sum_{i=1}^{n} x_{j i}
\end{aligned}
$$

Para conocer la importancia del nodo en relación con otros, se calcula el volumen de asociación a partir del número de enlaces; a este cálculo se le conoce como centralidad de grado. Esta medida es la más simple $\mathrm{y}$ mide el número de conexiones que un nodo tiene con otros. Al nodo que tiene la mayor cantidad de vecinos se le conoce como "hub". La centralidad de grado es un indicador local y estático, que sólo considera a los vecinos directos de un nodo.

De esta forma, para establecer la medida de centralidad de grado se calcula la matriz de adyacencia mostrada en la ecuación 8 (Wasserman y Faust, 1994).

Como se ha dicho, el grado de centralidad de un nodo $i$ representa el número de vínculos que mantiene. La centralidad de cada agente depende del tamaño de la red, donde la centralidad máxima se encuentra determinada por $n-1$ (Michael y Battiston, 2009).

\section{RESULTADOS}

El primer paso de la exploración metodológica consiste en analizar las grandes tendencias de crecimiento del empleo manufacturero a nivel mundial.

El Cuadro 1 muestra una comparativa del crecimiento del empleo a nivel de gran sector ISIC. Se observa una ralentización de la totalidad de las actividades económicas, misma que anticipaba una potencial crisis económica, incluso antes de la pandemia de 2020.

A pesar de ese menor dinamismo, hay actividades que crecen más que el resto, como las financieras y de seguros, mientras que las que sufren una mayor pérdida de dinamismo son las manufacturas. El análisis de los datos muestra algunas tendencias generales que es necesario entender. La primera de ellas es una pronunciada disminución en 
la capacidad de las economías para generar empleos. La tasa de crecimiento del empleo en el mundo se redujo de $0.7 \%$, en 2015 , a $0.2 \%$, en 2019. Existen algunas actividades en las que esa disminución es aún más pronunciada, como en el caso de las manufacturas. Esa reducción es un síntoma inequívoco de la desaceleración en la economía mundial que se venía gestando antes de la crisis generada por la Covid-19.

CUADRO 1

CRecimiento del EMPLeO MUNDIAL POR ACTIVIDAD ISIC, 2015-2019

\begin{tabular}{l|c:c} 
Gran Actividad ISIC & Crecimiento 2015 & Crecimiento 2019 \\
\hline Actividades financieras y de seguros & $1.4 \%$ & $0.7 \%$ \\
Arte, entretenimiento y recreación & $0.8 \%$ & $0.4 \%$ \\
Minas y canteras & $0.0 \%$ & $0.3 \%$ \\
Información y comunicación & $0.7 \%$ & $0.2 \%$ \\
Manufactura & $1.2 \%$ & $0.2 \%$ \\
Act. Profesionales científicas y técnicas & $0.2 \%$ & $-0.1 \%$ \\
\hline Total general & $\mathbf{0 . 7 \%}$ & $\mathbf{0 . 2} \%$ \\
\hline \hline
\end{tabular}

Fuente: elaborado con datos del Banco Mundial y LinkedIn, Digital Data for Development.

No obstante, si se desea observar la modificación en los patrones de crecimiento generados por un mayor dinamismo laboral, y las modificaciones que ello crea en la migración del empleo, conviene desagregar las actividades para analizar la migración de talento intrasectorial.

Como se puede apreciar en el Cuadro 2, hay una tendencia generalizada de disminución del crecimiento del empleo que ha provocado de manera directa al menos dos fenómenos; por un lado, un cambio en la composición del empleo entre sectores, por lo que aquellos de mayor dinamismo se convirtieron en polos atractores del empleo expulsado por aquellos de mayor ralentización. Por el otro, una redistribución del empleo entre las ramas que componen los sectores.

$\mathrm{Al}$ interior del sector manufacturero se puede apreciar una considerable disminución del empleo en términos agregados, que incluso llega a ser negativa para el caso de actividades como la automotriz, los textiles, la manufactura eléctrica y electrónica, y la impresión, lo que en otros términos implica que esas actividades representan polos expulsores de empleo dentro del sector manufacturero. 
CUADRO 2

MANUFACTURA

\begin{tabular}{l|c|c|}
\hline \multicolumn{1}{|c}{ Industria } & Crecimiento 2015 & Crecimiento 2019 \\
\hline Energía renovable y medio ambiente & $3.3 \%$ & $1.8 \%$ \\
Fabricación de ferrocarriles & $1.6 \%$ & $0.9 \%$ \\
Aviación y aeroespacial & $3.5 \%$ & $0.7 \%$ \\
Automatización industrial & $1.6 \%$ & $0.6 \%$ \\
Empaques y contenedores & $0.6 \%$ & $0.4 \%$ \\
Farmacéuticos & $1.5 \%$ & $0.3 \%$ \\
Productos de papel y forestales & $-0.1 \%$ & $0.2 \%$ \\
Producción de alimentos & $1.8 \%$ & $0.2 \%$ \\
Maquinaria & $1.2 \%$ & $0.2 \%$ \\
Construcción naval & $0.4 \%$ & $0.0 \%$ \\
Químicos & $1.0 \%$ & $0.0 \%$ \\
Vidrio, cerámica y hormigón & $0.5 \%$ & $0.0 \%$ \\
Plásticos & $1.1 \%$ & $-0.1 \%$ \\
Automotriz & $0.6 \%$ & $-0.2 \%$ \\
Textiles & $1.1 \%$ & $-0.2 \%$ \\
Manufactura eléctrica y electrónica & $-0.5 \%$ & $-0.5 \%$ \\
Impresión & $0.1 \%$ & $-0.7 \%$ \\
\hline Total general & $1.2 \%$ & $0.2 \%$ \\
\hline \hline
\end{tabular}

Fuente: elaborado con datos del Banco Mundial y LinkedIn, Digital Data for Development.

Para comprender las tendencias generadas no sólo a partir del crecimiento del empleo, sino también la migración inter e intra sectorial que se produce como resultado del cambio tecnológico, es necesario incorporar la variable de migración de talento. El segundo paso de la aplicación metodológica consiste en el uso de teoría de grafos a los datos a través del software Sytoscape. Ello permite analizar las grandes tendencias de los flujos de talento al interior y exterior de la industria manufacturera y responder a las preguntas: ¿hacia qué ramas de a manufactura se está moviendo el talento? ¿Es el crecimiento del empleo el que determina los flujos de migración de talento, o más bien es la prevalencia de habilidades la que influye en esa migración?

El grafo de la Figura 3 muestra los flujos de migración de talento en la manufactura mundial y mide las siguientes dimensiones: la primera se encuentra constituida por los nodos, que representan la rama de la manufactura hacia la cual se mueven los flujos de talento. El tamaño del 
nodo o círculo mide la tasa de crecimiento del empleo de esa actividad en los últimos 5 años. El color del nodo indica si el crecimiento fue positivo o negativo. Por ejemplo, en el caso de la rama Impresión, el color rojo del nodo indica una tasa de crecimiento promedio del empleo de 2015 a 2019 de -0.7 por ciento. El color verde del nodo de la industria farmacéutica indica que su crecimiento durante el mismo periodo fue positivo, y su tamaño muestra una tasa de crecimiento promedio de 0.3 por ciento.

La segunda dimensión se constituye por los enlaces, que miden la migración de talento de cada una de las ramas. El grosor del enlace indica la magnitud del flujo, mientras que la tonalidad del enlace es indicador de la dirección del flujo, es decir, si es positivo en términos netos, o negativo. Por ejemplo, el enlace de la rama de Energías renovables y medio ambiente indica que un flujo importante de talento de la manufactura se mueve hacia esa rama y su color verde indica que ese flujo es positivo; el grosor del enlace indica la magnitud del flujo, que en ese caso es de 1571.000 trabajadores (las cifras se encuentran en miles). Por su parte, el enlace de la rama Automotriz muestra que el sector fue expulsor neto de talento en el mismo periodo, con una cantidad de 855000 trabajadores.

\section{FIGURA 3}

GRAFO DE LA MIGRACIÓN DE TALENTO EN LA MANUFACTURA MUNDIAL, 2015-2019

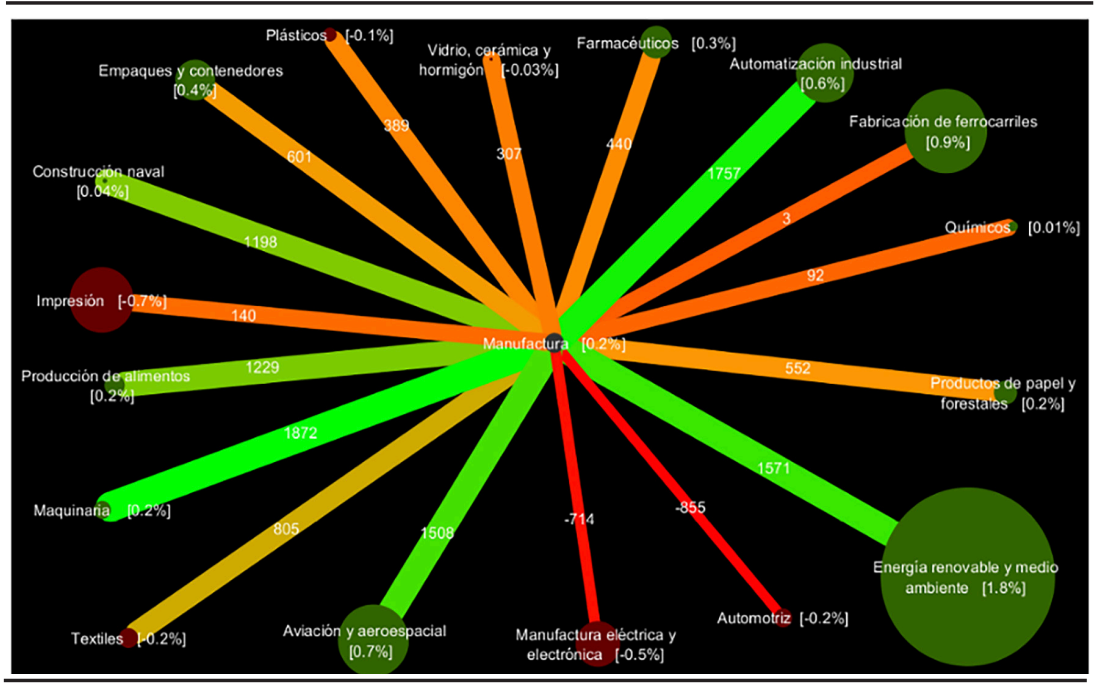

Fuente: elaborada con datos de Banco Mundial y LinkedIn, Digital Data for Development. 
De esta forma, el grafo sintetiza una gran cantidad de información, tanto de las tasas de crecimiento del empleo en la industria, como de los flujos de talento de las ramas que la componen.

Dos tendencias importantes se muestran en el grafo 3. Por un lado, un conjunto de ramas de la manufactura que no presentan crecimiento del empleo en los últimos 5 años, hecho que indica una tendencia de estancamiento de esas actividades industriales. Este grupo se encuentra constituido por las ramas: industria automotriz, manufactura eléctrica y electrónica, textiles e impresión.

En el extremo opuesto se muestran las ramas más dinámicas de la industria, buena parte de ellas muy alineadas con dos tendencias mundiales recientes; por un lado, la del cuidado del medio ambiente $\mathrm{y}$, por el otro, procesos de automatización ligados a la industria 4.0. Estas ramas de alto crecimiento del empleo en el último quinquenio son: energías renovables y medio ambiente, empaques y contenedores, fármacos, fabricación de ferrocarriles, automatización industrial y aviación y aeroespacial.

En medio de estos dos grupos se encuentran ramas manufactureras con crecimientos anuales que alternan entre positivos y negativos, pero que en promedio oscilan en torno a valores muy cercanos a cero, por lo que se puede hablar de una suerte de estado estacionario en estas actividades. Este grupo se encuentra constituido por las ramas: productos de papel, químicos, vidrio y cerámica, plásticos, construcción naval y maquinaria.

Sin embargo, para tener un panorama mucho más certero de las tendencias de largo plazo, es necesario incluir la migración de talento entre las ramas. Ello permite analizar no sólo las de alto crecimiento del empleo, sino también hacia dónde se está moviendo el talento en la manufactura mundial.

Cuando se analiza de esta manera se observan tres grandes ramas que dirigen la tendencia en la manufactura mundial; la primera es la de Energía renovable y medio ambiente, que no sólo es la rama de mayor crecimiento, sino también la de mayor atracción de talento en la manufactura mundial, como muestra el grosor de su enlace; anualmente, poco más de 1,500.000 trabajadores migran hacia esa actividad.

El segundo gran polo de atracción se encuentra constituido por la Automatización industrial, que muestra no sólo elevadas tasas de 
crecimiento del empleo, sino que anualmente incorpora poco más de 1,700.000 nuevos talentos. Esta rama se encuentra estrechamente relacionada con la industria 4.0.

Por último, el tercer gran polo de crecimiento y atracción de talento dentro de la manufactura es la industria de Aviación y aeroespacial. Y es probable que esta tendencia continúe, ya que existen razones que fundamentan una perspectiva de crecimiento importante de esta industria durante los próximos años, como el crecimiento de las megacuidades, el crecimiento de la clase media global y la mayor carga tecnológica y de servicios digitales de entretenimiento que se incorporan cada vez en mayor proporción en las aeronaves (Airbus, 2018).

En síntesis, el análisis de grafos muestra dos grandes actividades atractoras netas de talento y que no sólo han marcado la tendencia de la manufactura mundial en los últimos 5 años, sino que se espera que continúen dirigiendo el talento en los próximos años y, por lo tanto, las habilidades requeridas en el futuro. Estas actividades son el cuidado del medio ambiente y los procesos de automatización industrial (industria 4.0).

Es de interés de este artículo comparar las tendencias en la manufactura mundial con lo ocurrido en el mismo sector en México. Ello permitirá, en principio, arrojar luz sobre las tendencias que presenta la manufactura en México y hasta qué punto éstas se corresponden con las analizadas a nivel mundial.

La Figura 4 muestra la red de migración de talento en la industria manufacturera en México. En ella se pueden observar algunas tendencias que contrastan con lo ocurrido a nivel mundial; por un lado, prácticamente la totalidad de las ramas industriales en México muestran un crecimiento negativo en el volumen de empleo y una importante migración de talento. Por otro lado, la pérdida de empleo del sector manufacturero en México es, por mucho, más pronunciada que en el caso de la industria mundial.

De manera específica, son las ramas del sector automotriz, la manufactura electrónica, los químicos, la producción de alimentos y la automatización industrial las que sufren la mayor pérdida de empleo en los últimos 5 años. Sin embargo, dentro de estos sectores, el de Automatización industrial y las actividades vinculadas a la industria 4.0 siguen patrones diferentes en México y en el mundo. Mientras que para el 
primer caso es una industria expulsora de empleo, en el mundo muestra un dinamismo muy importante.

Cabe señalar que durante el periodo de estudio las ramas de la industria automotriz, manufactura eléctrica y electrónica muestran una gran pérdida de empleo y flujos negativos de talento que son expulsados hacia otras actividades, tanto a otras ramas al interior de las manufacturas como fuera de ellas.

\section{FIGURA 4}

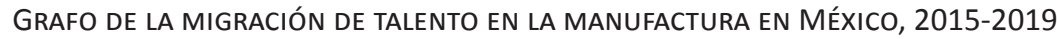

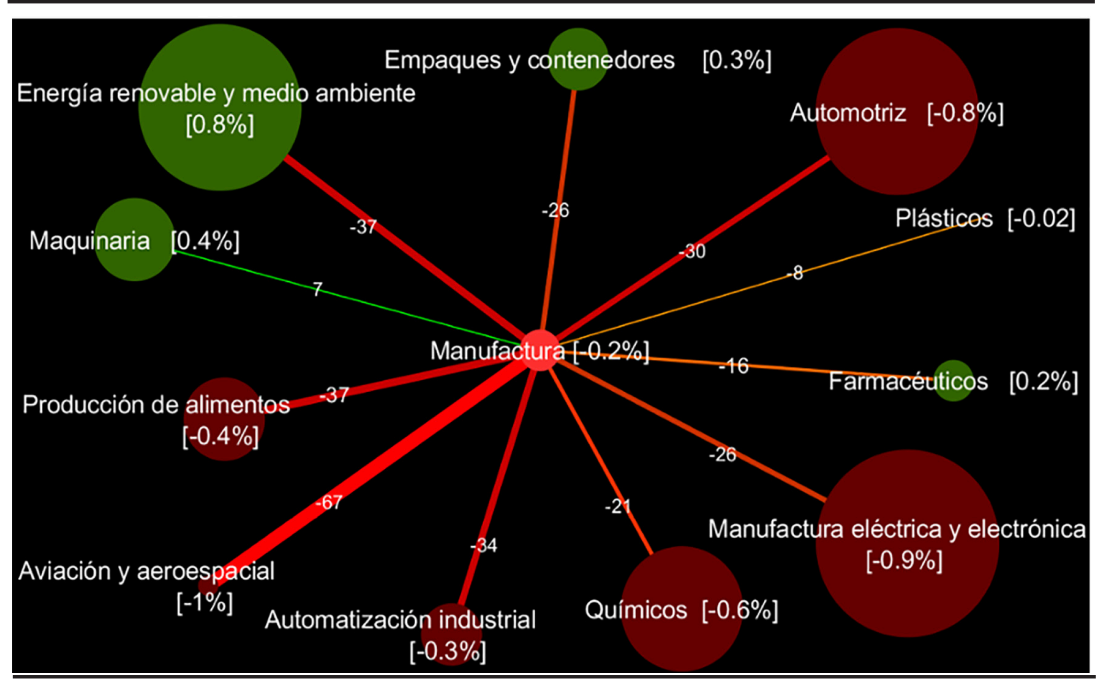

Fuente: elaborado con datos del Banco Mundial y Linkedln, Digital Data for Development.

Por su parte, una tendencia que se valida tanto en el mundo como en México es el alto crecimiento de las actividades vinculadas con la preservación del medio ambiente y el cuidado del planeta; ello se observa en el alto crecimiento relativo de las ramas Energías renovables y medio ambiente, y Empaques y contenedores. Sin embargo, los enlaces en ambos casos muestran que, a pesar del alto crecimiento, hay una salida neta de talento del orden de 37000 y 26000 trabajadores, respetivamente, en el periodo 2015-2019.5 Lo anterior indica que, a pesar del dinamismo de esas ramas, no logran retener talento.

La única rama que muestra un crecimiento moderado y que en términos netos es atractora de talento es la de Maquinaria.

$5 \operatorname{ILOS}(221) .<$ https://ilostat.ilo.org/>. 
Lo anterior muestra un hecho relevante, y a la vez preocupante, que quizá contribuya a explicar el muy bajo crecimiento de la economía mexicana durante el periodo de estudio, y es que por 5 años consecutivos el sector manufacturero ${ }^{6}$ en México ha experimentado un decrecimiento del empleo y una expulsión neta de talento, como se aprecia en el color rojo (deficitario de talento en términos netos) en 10 de sus 11 enlaces. Ello implica no sólo la incapacidad del sector en este periodo para generar empleos, sino también, y quizá más preocupante por sus implicaciones de largo plazo, una migración neta de talento hacia otras actividades económicas, quizá mejor remuneradas. Esto ocurre tanto en las ramas manufactureras de mayor tradición en México -la industria Automotriz- como en aquellas relativamente más jóvenes y de mayor empuje -el sector de Aviación y aeroespacial- y en aquellas que en el mundo representan la promesa de crecimiento de las manufacturas -la Automatización industrial y la manufactura eléctrica y electrónica-.

Por otra parte, al medir la centralidad de grado de las industrias manufactureras a nivel internacional y de las habilidades laborales requeridas, se obtienen los resultados resumidos en el Cuadro 3.

Las medidas de centralidad de grado permiten entender cuáles son las actividades que requieren una mayor cantidad de habilidades por parte de los trabajadores para desempeñarse en ellas (grado de salida o Outdegree), así como las principales habilidades demandadas por parte de las ramas (grado de entrada o Indegree).

Un número mayor de enlaces de salida indica las ramas dentro de la manufactura que requieren una mayor cantidad de habilidades para desempeñarse en ellas, mientras que un número mayor de enlaces de entrada, indica las habilidades más requeridas o demandas para trabajar dentro de la industria manufacturera en su conjunto.

6 Huelga decir que, en términos generales, las manufacturas constituyen el sector con la mayor capacidad de arrastre y encadenamientos productivos hacia atrás y hacia adelante. 
CUADRO 3

CENTRALIDAD DE GRADO DEL SECTOR MANUFACTURERO A NIVEL MUNDIAL

\begin{tabular}{|c|c|c|c|c|c|}
\hline & 2015 & 2019 & & 2015 & 2019 \\
\hline Industrias & $\begin{array}{c}\text { Grado } \\
\text { de Salida } \\
\text { (Outdegree) }\end{array}$ & $\begin{array}{c}\text { Grado } \\
\text { de Salida } \\
\text { (Outdegree) }\end{array}$ & Habilidades & $\begin{array}{l}\text { Grado de } \\
\text { Entrada } \\
\text { (Indegree) }\end{array}$ & $\begin{array}{c}\text { Grado de Entrada } \\
\text { (Indegree) }\end{array}$ \\
\hline Automotriz & 4 & 4 & Negocios & 14 & 12 \\
\hline $\begin{array}{c}\text { Aviación y } \\
\text { aeroespacial }\end{array}$ & 4 & 4 & $\begin{array}{c}\text { Tecnológicas } \\
\text { disruptivas }\end{array}$ & 5 & 3 \\
\hline Productos químicos & 5 & 4 & Blandas & 12 & 13 \\
\hline $\begin{array}{l}\text { Manufactura } \\
\text { eléctrica y } \\
\text { electrónica }\end{array}$ & 5 & 5 & $\begin{array}{l}\text { Industriales } \\
\text { especializadas }\end{array}$ & 14 & 14 \\
\hline $\begin{array}{l}\text { Producción de } \\
\text { alimentos }\end{array}$ & 4 & 4 & Tecnológicas & 12 & 14 \\
\hline $\begin{array}{c}\text { Automatización } \\
\text { industrial }\end{array}$ & 4 & 3 & $\begin{array}{c}\text { Nodos } \\
\text { disponibles }\end{array}$ & 14 & 14 \\
\hline Maquinaria & 4 & 4 & & & \\
\hline $\begin{array}{c}\text { Envases y } \\
\text { contenedores }\end{array}$ & 4 & 4 & & & \\
\hline $\begin{array}{l}\text { Productos de papel y } \\
\text { forestales }\end{array}$ & 4 & 4 & & & \\
\hline $\begin{array}{c}\text { Productos } \\
\text { farmacéuticos }\end{array}$ & 2 & 4 & & & \\
\hline Plásticos & 5 & 4 & & & \\
\hline Impresión & 4 & 4 & & & \\
\hline $\begin{array}{l}\text { Energía renovable y } \\
\text { medio ambiente }\end{array}$ & 4 & 4 & & & \\
\hline Textiles & 4 & 4 & & & \\
\hline Nodos disponibles & 5 & 5 & & & \\
\hline
\end{tabular}

Fuente: elaborado con datos del Banco Mundial y LinkedIn, Digital Data for Development.

Se observa que a nivel mundial no varía mucho la centralidad de grado respecto a las distintas industrias que conforman el sector manufacturero, con excepción de la elaboración de productos farmacéuticos, donde ha aumentado el número de habilidades para desempeñarse en ello. En cuanto a las habilidades requeridas por los trabajadores, ha disminuido la centralidad de las habilidades en negocios y en tecnologías disruptivas, mientras que aumentó en habilidades blandas y habilidades tecnológicas. Al presentar la información contenida en el Cuadro 3 en forma de grafos, se observa de manera más clara la evolución de las habilidades requeridas a nivel internacional en 2019 respecto a 2015. 
2015

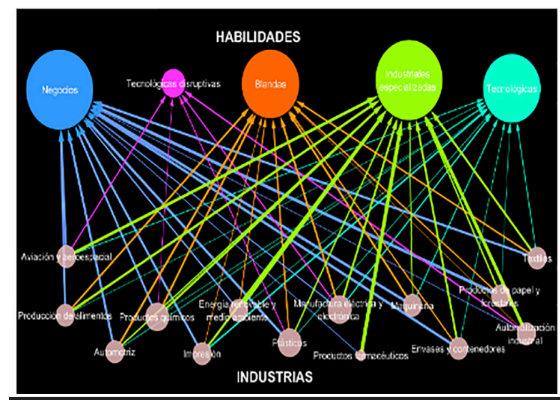

2019

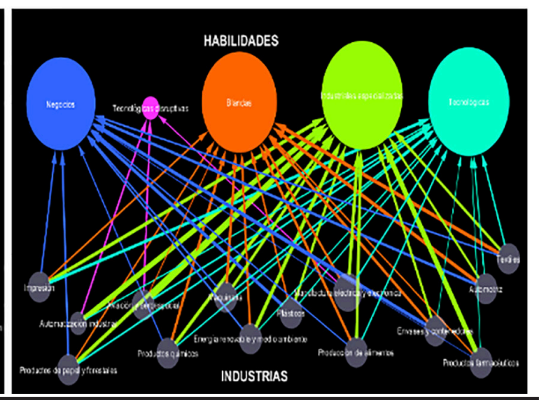

Fuente: elaborado con datos del Banco Mundial y LinkedIn, Digital Data for Development.

Esta información permite establecer un mapeo de las capacidades requeridas por parte del capital humano para desempeñarse dentro de la industria manufacturera, pero también, y quizá aún más importante, desde el punto de vista de la política pública ofrece la posibilidad de establecer un mapeo de las capacidades endógenas requeridas (en términos de programas de capacitación, oferta de carreras, planes de estudio, etc.) para "crear" y desarrollar los segmentos o ramas de la manufactura en las cuales México tiene una ventana de oportunidad para la inserción en las industrias que muestran un amplio dinamismo en términos de crecimiento y creación de empleo a nivel internacional, atractores netos de talento a nivel mundial.

Representa también un insumo para la toma de decisiones en torno a cuáles debieran ser los sectores prioritarios de desarrollo en función del crecimiento de habilidades a nivel internacional. Desde esta perspectiva, el mayor dinamismo representa una mayor oportunidad de negocios, así como una mayor probabilidad de obtener un empleo bien remunerado.

\section{CONCLUSIONES}

Este trabajo muestra la dinámica de migración laboral y atracción/ expulsión de talento en las manufacturas en el mundo y en México a partir de las habilidades requeridas por las distintas industrias a nivel 
mundial. Se observa una pérdida importante y constante del crecimiento del empleo en el mundo entre 2015 y 2019; sin embargo, esta tendencia es aún mayor en el caso de México.

Si bien hay un menor dinamismo manufacturero, también se observa una importante migración de talento de las actividades manufactureras tradicionales (sector automotriz, textiles e impresión) hacia actividades de más reciente aparición, como Energías renovables y medio ambiente, y Automatización industrial (industria 4.0).

Un hecho preocupante que revela esta investigación es que dicha migración al interior de las manufacturas a nivel mundial hacia actividades más rentables y de mayor futuro no existe en México, lo que comprueba indirectamente la pérdida de centralidad del sector en el sistema económico. Se verifica una pérdida generalizada del empleo industrial, con una salida de talento en 10 de las 11 actividades analizadas para la manufactura mexicana.

En el caso de México las actividades dinámicas y de muy alta atracción de talento en otras latitudes muestran salidas netas de talento durante el periodo de estudio, por lo que no se observan actividades que puedan representar polos visibles de recuperación de la dinámica industrial y, por lo tanto, de la totalidad de la actividad económica en México.

Lo anterior, sin duda alguna, es resultado y síntoma de la falta de una política industrial que oriente el quehacer de la actividad manufacturera en México hacia donde se anticipen resultados favorables. Mientras México pierde talento en estos sectores, otras economías lo están generando, y ello tendrá repercusiones en el mediano plazo, cuando otros países cuenten con sectores amplios de energías renovables e industrias automatizadas, y México quede rezagado para continuar las tendencias mostradas en este estudio.

Futuras líneas de investigación pueden analizar hacia dónde (qué sectores) y qué tipo de capacidades (habilidades de la fuerza laboral) son las que se expulsan del sector manufacturero en México.

\section{REFERENCIAS}

AirBus (2018), Global market Forecast: Global Networks, Global Citizens, France.

Antonelli, C. (2008), Localized technological change. Towards the economics of complexity, Londres, Routledge. 
Barro, R. y J. Lee (1993), "International comparisons of educational attainment", Journal of monetary economics, 32(3), pp. 363-394.

Becker, G. (1994), Human capital revisited. In Human Capital: A Theoretical and Empirical Analysis with Special Reference to Education, 3. ${ }^{\mathrm{a}} \mathrm{ed}$., EE. UU., The University of Chicago Press, pp. 15-28.

Cañibano, C. y J. Potts (2019) “Toward an evolutionary theory of human capital”, Journal of Evolutionary Economics, núm. 29, pp. 1017-1035.

Cohen, M.D. y D.A. Levinthal (1990), “Absorptive Capacity: A New Perspective on Learning and Innovation", Administrative Science Quarterly, núm. 35 , pp. 128-152.

Díaz, H., M. Morales y S. Cabrera (2019), “Dinámica de la cadena global aeroespacial: un análisis de teoría de grafos”, Contaduría y Administración, 65 (4), pp. 1-24.

Dopfer K., J. Foster y J. Potts (2004), “Micro-meso-macro”, Journal of Evolutionary Economics, núm. 14, pp. 263-279.

Dopfer, K. (2005), Evolutionary Foundations of Economics, New York, Cambridge University Press.

Dopfer, K. y J. Potts (2008), The General Theory of Economic Evolution, Londres y New York, Routledge.

Dosi, G., R.R. Nelson y S.G. Winter (eds.) (2000), The Nature and Dynamics of Organizational Capabilities, New York, Oxford University Press.

Dosi, G. y M. Cimoli (1994), "De los paradigmas tecnológicos a los sistemas nacionales de producción e innovación”, Comercio exterior, 44(8), pp. 669-82.

Dosi, G., M. Faillo y L. Marengo (2003), “Organizational Capabilities, Patterns of Knowledge Accumulation and Governance Structures in Business Firms. An Introduction", LEM, Working Paper Series, num 11, Sant'Anna School of Advanced Studies, Pisa.

Foss, N. (2006), "The Emerging Knowledge Governance Approach: Challenges and Characteristics", Working Paper, Danish Research Unit for Industrial Dynamics, núm. 06-10.

Fuentes, N. y A. García (2009), "Jerarquización sectorial de la economía mexicana: un enfoque de teoría de grafos", Problemas del Desarrollo. Revista Latinoamericana de Economía, 40(158), pp. 137-159

García, A., A. Morillas y C. Ramos (2005), "Relaciones interindustriales y difusión de la innovación. Una aproximación desde la teoría de redes”, Estadística española, 47 (160), pp. 475-499.

Harper, D. A. (2018), "Innovation and institutions from the bottom up: an introduction", Journal of Institutional Economics, Vol. 14, Edición especial 6, pp. 975-1001.

Hodgson, G.M. (2007), "Teorías evolucionistas de la empresa basadas en las competencias", en G.M. Hodgson, Economía institucional y evolutiva contemporánea, México, UAM, pp. 207-249 
Marengo, L. y C. Pasquali (2008). “El mercado, la empresa y la primacía del conocimiento", en R. Viale (comp.), Las nuevas economías. De la economía evolucionista a la economía cognitiva: más allá de las fallas de la teoría neoclásica, México, FLACSO, pp. 65-78.

Michael D.K. y S. Battiston (2009), "From Graph Theory to Models of Economic Networks. A Tutorial", en A.K. Naimzada, S. Stefani y A. Torriero (eds.), Networks, Topology and Dynamics. Lecture Notes in Economics and Mathematical Systems, vol. 613, Springer, Berlin, Heidelberg, pp 2363.

Nelson, R., G. Dossi, y C. Helfat (2018), Modern evolutionary economics: An overview, Cambridge University Press

Nelson, R. y S. Winter (1982), An evolutionary theory of economic change, EE. UU., Harvard University Press.

Newman, Mark (2010), Networks: an introduction. Oxford: University Press.

Patt, A., J. Ruhose, S. Wiederhold y M. Flores (2020), "International Emigrant Selection on Occupational Skills", Journal of the European Economic Association. Discussion Paper 10837. Bonn, IZA.

Rosenberg, N. (1982), Inside the Black Box, Inglaterra, Cambridge University Press.

Vromen, J. (2006a), “Organizational Routines and Individual Skills. Beyond Analogy", Erasmus Institute for Philosophy and Economics Working Papers.

Vromen, J. (2006b). "Routines, Genes and Program-Based Behavior”, Journal of Evolutionary Economics, núm. 16, pp. 543-560.

Wasserman, S. y L. Faust (1994), Social network analysis: Methods and applications. Cambridge: University Press."

Zhu, T.J., A. Fritzler, J.A.K. Orlowski (2018), "Data Insights: Jobs, Skills and Migration trends Methodology and Validation Results", World Bank Group. $<$ http://documents1.worldbank.org/curated/en/827991542143093021/ pdf/World-Bank-Group-LinkedIn-Data-Insights-Jobs-Skills-and-Migration-Trends-Methodology-and-Validation-Results.pdf $>$. 